ESAIM: PROCEEDINGS, August 2008, Vol. 24, p. 34-45

C. Dobrzynski, P. Frey, Ph. Pebay, Editors

\title{
LOCAL TOPOLOGICAL MODIFICATION OF HEXAHEDRAL MESHES PART II: COMBINATORICS AND RELATION TO BOY SURFACE
}

\author{
Katerina Jurkova ${ }^{1}$, Franck Ledoux ${ }^{2}$, Raphael Kuate ${ }^{3}$, Tom Rickmeyer ${ }^{4}$, \\ Timothy J. TAUTGES ${ }^{5}$ AND Hamdi Zorgati ${ }^{6}$
}

\begin{abstract}
Hexahedral meshes are structured as a set of ordered layer of hexes which makes local topological modifications difficult to do. For instance, removing an hex generally implies to remove a complete layer of hexes. Few works focus on local topological modifications in hexahedral meshes. In this paper, we provide some results which extend and complete some existing works $[1,14,15,17]$, proving in a first part that the flipping operations defined by M. BERN and D. EPPSTEIN are combinatorially free and showing in a second part how to introduce a Boy surface into a dual mesh. This operation allows us to modify the parity of the number of hexes in the primal mesh, thing that can not be done by the M. BERN and D. EPPSTEIn basis of operations.

Résumé. Tout maillage hexaédrique est structuré comme un ensemble ordonné de couches de mailles. Cette structuration rend difficile les modifications topologiques locales du maillage. Par exemple, retirer une maille du maillage nécessite souvent le retrait d'une couche complète de mailles. Peu de travaux s'intéressent à ce problème. Dans ce papier, nous donnons différents résultats qui étendent et complètent des travaux existants $[1,14,15,17]$ prouvant dans une première partie que les opérations de flipping définies par M. BERN et D. EPPSTEIN sont libres (au sens combinatoire) et montrant dans une seconde partie comment introduire concrètement une surface de Boy dans un maillage dual d'un maillage hexaédrique. Ceci permet de modifier la parité du nombre de mailles contenues dans le maillage primal, ce qui n'est pas possible avec la base d'opérations de M. BERN et D. EPPSTEIN.
\end{abstract}

\section{INTRODUCTION}

Local topological modification of hexahedral meshes has been considered difficult due to the global structure of hexahedral meshes. Indeed, contrary to tetrahedral meshes, hexahedral meshes are structured as a set of ordered layers of hexes which makes local topological modifications difficult to perform. For instance, if we want to remove a hex from a hexahedral mesh, it is generally necessary to remove a complete layer of hexes at least. This topological structure of a hexahedral mesh is known as the Spatial Twist Continuum [11] which defines the topology of the dual of hexahedral mesh.

1 NTI FM TUL, Hálkova 6, 46117 Liberec 1, Czech Republic; e-mail: katerina.jurkova@tul.cz

${ }^{2}$ CEA/DAM - Île-de-France, Bruyères-Le-Châtel, France; e-mail: franck.ledoux@cea.fr

${ }^{3}$ Laboratoire Jacques-Louis Lions, Université Pierre et Marie Curie, Paris, France; e-mail: kuate@ann.jussieu.fr

${ }^{4}$ University of Wisconsin-Madison, Madison, U.S.A.; e-mail: tom.rickmeyer@gmail.com

${ }^{5}$ Argonne National Laboratory, Mathematics and Computer Science Div., Madison, U.S.A.; e-mail: tautges@mcs.anl.gov

${ }^{6}$ Faculté des Sciences de Tunis, Université Tunis El Manar 2092, Tunisia; e-mail: hamdi.zorgati@fst.rnu.tn

(C) EDP Sciences, SMAI 2008 
Few works focus on the necessary issue of the local topological modifications of hexahedral meshes. This is in contrast with tetrahedral meshes and quadrilateral meshes [4] where local topological modifications are crucial to ensure the robustness of mesh generation algorithms. Local topological modification is often treated peripherally in the development of hex mesh generation algorithms $[3,5,9,13,16]$. In [1], M. BERN and D. EPPSTEIN describe a set flipping operations for hexahedral meshes. These flipping operations are analogue to the well-known flipping operations for simplicial meshes. In $[14,15,17]$, the authors attempt to define a complete set of local atomic modification operations. By complete they mean that we can transform a given hexahedral mesh into any other ones with the same quad boundary mesh. By local they mean that the connectivity altered by an operation is local to a few hexes and not to complete layer of hexes. By atomic they mean that each operation must be irreducible, that is can not be done as a sequence of others operations. These operations are simpler, more local and more fundamental that the flipping operations defined by M. BERN and D. EPPSTEIN.

The final goal of the authors of $[14,15,17]$ has not been achieved and the work described here continues it. Several issues remain unsolved:

- Each flipping operation defined by M. BERN and D. EPPSTEIn should be decomposed as a combination of atomic operations. This task is done in a joint paper [18] which was the starting point of this work.

- Defining a complete set of atomic operations is equivalent to define a set of atomic operations which should be a basis of the hexahedral mesh modification operations. This task is essential and needs a deep theoretical work in combinatorial topology. We have not proved that the set of operations defined in this paper is a basis. But, in Section 3, the combinatorial freedom of the flipping operations defined by M. BERN and D. EPPSTEIN is proved.

- Each of the flipping operations and each of the atomic operations preserve the parity (odd or even) of the number of hexes in the mesh. Thus to be able to modify any hex mesh into another one, at least one operation is missing. In order to change the parity of the number of hexes in a mesh, a new surface can be introduced in the dual of this mesh in such a way that the number of dual vertices increases by an odd number. A suitable candidate is the Boy surface. The hypothesis of introducing a Boy surface in the dual of a hex mesh was done by M. Bern and D. Eppstein [1]. In Section 4, we propose a constructive way to introduce this surface into the dual mesh.

In addition, a soft (Figure 4) permitting the computing and the visualisation of the hex mesh dual has been implemented. It can duplicate all of M. BERN and D. EPPSTEIN operations using the atomic operations.

We begin this paper by introducing some basic terms that will be used along the paper.

\section{Basic Terminology}

In this section, we gather usual definitions we can find in algebraic geometry or topological geometry. For more details about polytope theory, we refer to $[2,6-8,19]$. We also refer to [12] for an interesting introduction to these concepts.

\subsection{Generalities about Polytopes}

Definition 2.1. A polytope $P \subseteq \mathbb{R}^{d}$ is the convex hull of a finite number of points in $\mathbb{R}^{d}$. A polytope can also be defined as the bounded intersection of finitely many closed halfspaces $H_{+}=\left\{\mathbf{x} \in \mathbb{R}^{d}: \mathbf{a}^{T} \mathbf{x} \geq a_{0}\right\}$ where $a_{0} \in \mathbb{R}$ and $\mathbf{a}^{T} \in \mathbb{R}^{d}$.

The dimension $\operatorname{dim}(P)$ of a polytope is the dimension of its affine hull. A $d$-dimensional polytope is referred as a $d$-polytope.

Definition 2.2. $A$ face of a polytope $P$ is a subset $F \subseteq P$ of the form $F=P \cap\left\{\mathbf{x} \in \mathbb{R}^{d}: \mathbf{a}^{T} \mathbf{x}=a_{0}\right\}$ where $\mathbf{a}^{T} \mathbf{x} \geq a_{0}$ is a valid inequality for $P$.

Also the dimension of a face is the dimension of its affine hull. A $k$-dimensional face is called a $k$-face. A facet of a polytope $P$ is a $(\operatorname{dim}(P)-1)$-face. 
Definition 2.3. A simple d-polytope is one whose $k$-faces are formed by the intersection of $(d-k)$ facets with $0 \leq k \leq d$.

For instance, if we consider a 3 -polytope, each 0 -face is formed by the intersection of $3-0=3$ facets, i.e. faces or 2-faces, and each 1-face is formed by the intersection of $3-1=2$ facets. If we consider a single isolated polytope (not contained in a complex of polytopes), a simple $d$-polytope is a $d$-polytope whose vertices are $d$-valent.

Definition 2.4. A combinatorial d-cube is a d-polytope combinatorially equivalent to the standard d-cube (or regular $d$-cube) $Q^{d}=[-1,1]^{d}$.

Definition 2.5. A d-polytope is cubical if all its facets are combinatorial cubes.

Note that, for $d \geq 3$, every simple cubical $d$-polytope is a combinatorial $d$-cube.

\subsection{Polytopal Complexes and Meshes}

Definition 2.6. A polytopal complex, or a complex of polytopes, $C$ is a finite set of polytopes (called faces or cells) in $\mathbb{R}^{n}, n \geq 0$, that contains all the faces of its cells and that satisfies the intersection property, that is, the intersection of any two cells $P, Q \in C$ is a face of both.

This definition implies that $\emptyset$ is contained in $C$ and each face $F$ of a cell $P \in C$ is also contained in $C$. The cells of dimension $k$ of a $d$-dimensional complex $C$ are called $k$-faces or $k$-cells. The cells of dimension $0,1, \mathrm{~d}-1$ and $\mathrm{d}$ are respectively called the vertices, the edges, the ridges and the facets.

Definition 2.7. A d-dimensional geometric mesh is a d-dimensional polytopal complex.

A hexahedral mesh is a particular case where the polytopes are combinatorial cubes. In other words, a hexahedral mesh is a 3 -dimensional cubical complex.

Remark: We do not provide here the definition of a topological mesh from a topological algebraic point of view. Indeed, this definition needs the knowledge of the CW complex which is out of the scope of this document. CW stands for closure-finite weak topology.

\subsection{Dual of Hexahedral Meshes}

The dual of a hexahedral mesh can be described in terms of polytopal complexes and arrangement of surfaces.

Definition 2.8. Consider a hexahedral mesh $M$ and denote by $M_{i}=\left\{M_{i}^{k} \mid 1 \leq k \leq p\right\}$ the set of the $i$-cells contained in $M$, where $p$ is their number and $0 \leq i \leq 3$. The dual of $M$ is defined as a one-to-one mapping $\psi: M \rightarrow M^{*}$ which preserves but reverses incidence relations:

$$
M_{i}^{k} \subseteq M_{j}^{k^{\prime}} \leftrightarrow \psi\left(M_{j}^{k^{\prime}}\right) \subseteq \psi\left(M_{i}^{k}\right) \text { with } 0 \leq i<j \leq 3 .
$$

The dual $M^{*}$ of a hexahedral mesh $M$ is a polytopal complex, such that each $k$-cell of $M^{*}$ is the dual of a $(3-k)$-cell of $M$. In the relation between $M$ and $M^{*}$, the mesh $M$ is also called the primal mesh. Note that the dual of a hexahedral mesh is in fact a complex of simple polytopes and can be seen as a simple arrangement of hyperplans [17].

\section{Combinatorial Freedom of Flipping Operations}

In this section, we describe the flipping operations defined by M. BERN and D. EPpstein in [1]. Note that Figures 1 and 2 are the same as those in [1].

Consider the well-known flipping operation for a triangular mesh, which switches one diagonal for the other in a convex quadrilateral $Q$ formed by two triangular elements sharing a side. We can think of this 2-2 flip (two elements in and two elements out) as exchanging the upper and lower facets of a three-dimensional simplex $P$ that projects to $Q$. 
The almost-as-well-known flips for tetrahedral meshes can be defined analogously by the exchange of the upper and lower facets of a four-dimensional simplex. The flips include the 2-3 flip, which exchanges two tetrahedra sharing a face for three sharing an edge, and the 1-4 flip which subdivides a tetrahedron by adding an interior vertex, along with their reverse flips.

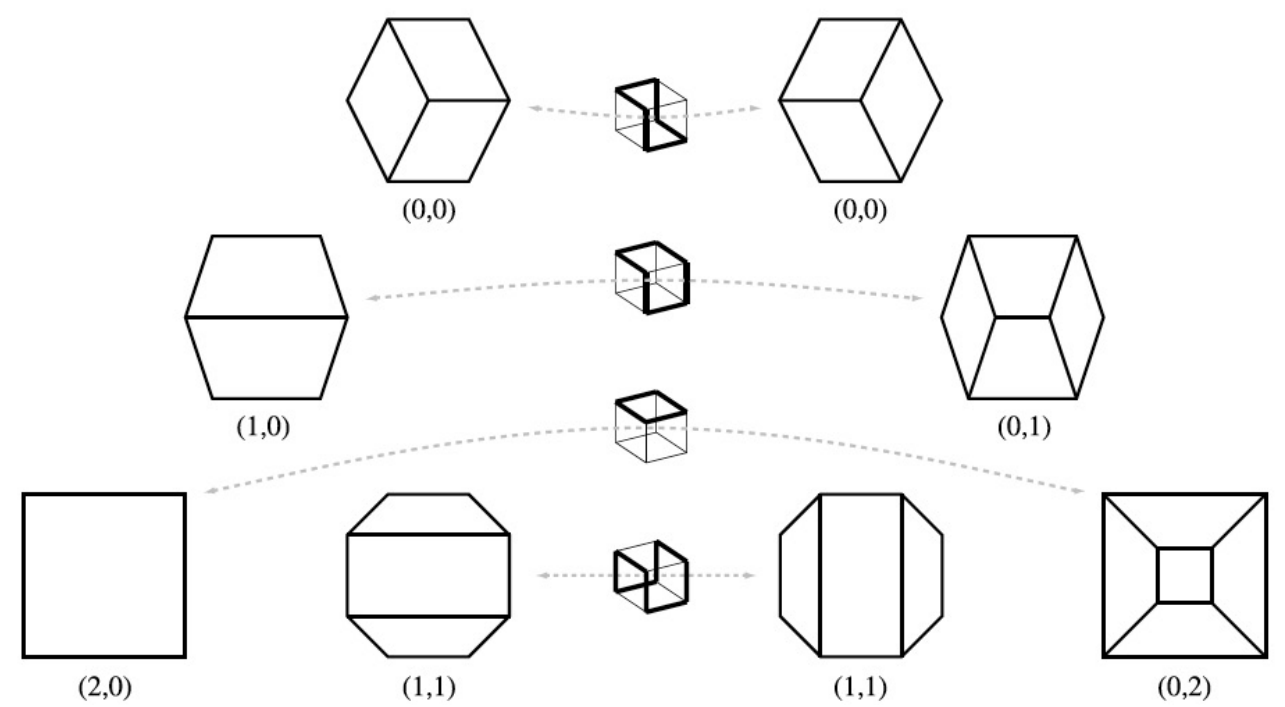

FIGURE 1. The flipping operations for quad meshes

This view of flipping extends to quad and hex meshes. The flips for quad meshes are induced by the combinatorially distinct exchanges of upper and lower facets of a three-dimensional combinatorial cube, as shown in Figure 1, and the flips for hexahedral meshes are induced by the combinatorially distinct exchanges of upper and lower facets of a four-dimensional hypercube, as shown in Figure 2. The facets of a $d$-dimensional cube or hypercube can be grouped into $d$ opposite pairs. In order to discover all possible flips, any set of facets of a cube or hypercube is described by how it relates to these pairs. A pair of integers $(X, Y)$ is assigned to any set $S$ of cube or hypercube facets: $X$ denotes the number of pairs of facets eXcluded from $S$, and $Y$ denotes the number of pairs of facets Yncluded in $S$. The remaining $d-X-Y$ pairs of facets have one of their two facets included in $S$, and the other facets excluded from $S$. The symmetries of the hypercube include all permutations of opposite pairs of facets, and all swaps of the two facets in each pair, so any two sets $S$ described by the same pair $(X, Y)$ are combinatorially equivalent. The meshes in Figure 1 and 2 are labeled by these pairs of numbers. If a set $S$ is represented by a pair $(X, Y)$, the complementary set is represented by pair $(Y, X)$. So, a flip can be described as the replacement of a set of mesh cells combinatorially equivalent to the set $(X, Y)$ by a different set of cells combinatorially equivalent to $(Y, X)$. In order to find all possible flips, it remains only to determine which pairs $(X, Y)$ form topological disks.

Theorem 3.1. Each set of flipping transformations represented in Figures 1 and 2 is topologically free, that is, we can not get one transformation using a combination of other ones.

Proof. Our approach to prove this theorem is purely algebraic. We denote by $n_{e}$ the number of new internal edges without a boundary vertex, $n_{v}$ the number of internal vertices, $n_{f}$ the number of internal faces without any boundary edge, $n_{h}$ the number of hexes in $3 \mathrm{D}$ and $n_{q}$ the number of quads in $2 \mathrm{D}$, created or removed through each transformation. We model them as follows :

$(0,0) \longleftrightarrow(0,0) \Longrightarrow \pm\left(0_{h}, 0_{f}, 0_{e}, 0_{v}\right)$

$(1,1) \longleftrightarrow(1,1) \Longrightarrow \pm\left(0_{h}, 0_{f}, 0_{e}, 0_{v}\right)$ 


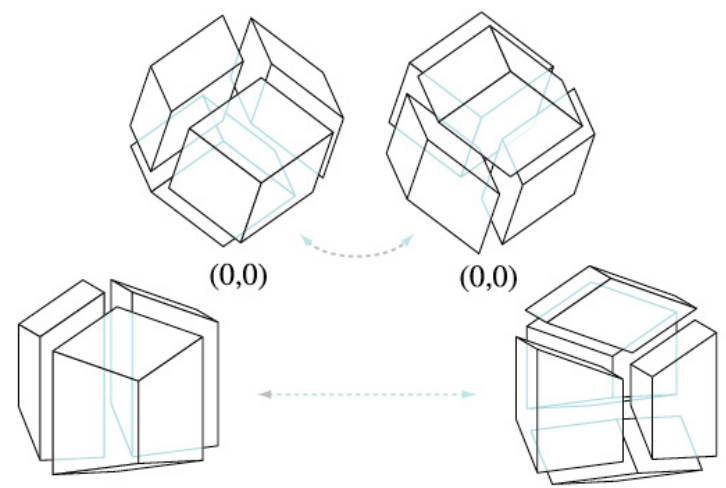

$(1,0)$

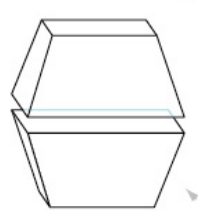

$(2,0)$

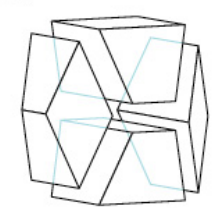

$(1,1)$
$(0,1)$

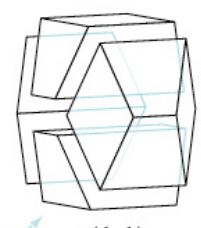

$(1,1)$

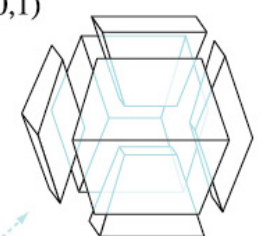

$(0,2)$

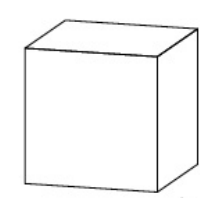

$(3,0)$

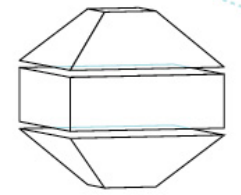

$(2,1)$

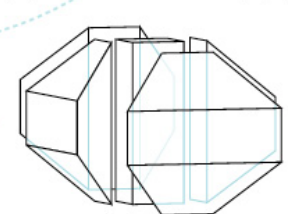

$(1,2)$

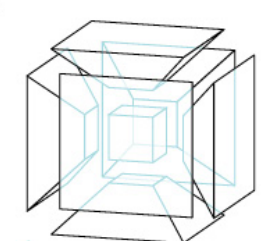

$(0,3)$

Figure 2. The flipping operations for hex meshes

$$
\begin{aligned}
& (1,0) \longleftrightarrow(0,1) \quad \Longrightarrow \quad \pm\left(2_{h}, 0_{f}, 1_{e}, 2_{v}\right) \\
& (2,0) \longleftrightarrow(0,2) \quad \Longrightarrow \pm\left(4_{h}, 1_{f}, 4_{e}, 4_{v}\right) \\
& (3,0) \longleftrightarrow(0,3) \quad \Longrightarrow \pm\left(6_{h}, 6_{f}, 12_{e}, 8_{v}\right) \\
& (2,1) \longleftrightarrow(1,2) \quad \Longrightarrow \pm\left(2_{h}, 0_{f}, 0_{e}, 0_{v}\right) \quad \text { in } 3 \mathrm{D}, \\
& (0,0) \longleftrightarrow(0,0) \quad \Longrightarrow \pm\left(0_{q}, 0_{e}, 0_{v}\right) \\
& (1,1) \longleftrightarrow(1,1) \quad \Longrightarrow \pm\left(0_{q}, 0_{e}, 0_{v}\right) \\
& (1,0) \longleftrightarrow(0,1) \quad \Longrightarrow \pm\left(2_{q}, 1_{e}, 2_{v}\right) \\
& (2,0) \longleftrightarrow(0,2) \quad \Longrightarrow \pm\left(4_{q}, 4_{e}, 4_{v}\right) \quad \text { in } 2 \mathrm{D} .
\end{aligned}
$$

Thus, if each set of flipping operations is not free, we can write one of the right member of the previous relationships as a linear combination of some others with coefficients in $\mathbb{Z}$. Let us look at these operations without $(0,0) \longleftrightarrow(0,0)$ and $(1,1) \longleftrightarrow(1,1)$ because those do not change numerically anything ; we have to solve the following equations in $\mathbb{Z}$ for each of the unknowns fixed at 1 or -1 (for example $x=1$ means that we are going from the $(1,0)$ to the $(0,1)$ configuration and $x=-1$ is for the $(0,1)$ to $(1,0)$ one).

$$
\left\{\begin{array}{c}
x+2 y+3 z+t=0 \\
x+6 y=0 \\
x+4 y+12 z=0 \\
x+2 y+4 z=0
\end{array}\right.
$$


for the hexes flipping,

$$
\left\{\begin{array}{l}
x+2 y=0 \\
x+4 y=0
\end{array}\right.
$$

for the quads flipping.

These two systems have $x=y=z=t=0$ as solutions. It means that we can not write none of these operations as a combination of some of the others. Thus, the set of operations without $(0,0) \longleftrightarrow(0,0)$ and $(1,1) \longleftrightarrow$ $(1,1)$ is free. If we add these two operations, it will neither change the equations or their solutions. The possible solutions of these system could have been considered as result of one of these two operations when combining all the others, thus we can conclude that each set is topologically free.

\section{Changing Parity by introducing a Boy Surface into an Arrangement of SuRFACES}

There exists at least one missing transformation in the set of flipping operations and in the atomic operations defined in [18] since they can not change the parity of the number of hexes, thing that could occur in reality. In order to change the parity of the number of hexes in a mesh, a new surface can be introduced into the dual of this mesh in such a way that the number of dual vertices increases by an odd number. A suitable candidate is the Boy surface which is a projective plane embedded in $\mathbb{R}^{3}$ in such a way that there is one triple intersection point, connected to itself by double-intersection curves in a pattern of three self-loops (see Figure 3). Note that the resulting arrangement of surfaces may have some self-loops and multiple adjacencies, but we can likely remove them by pillowing without further changes of parity.

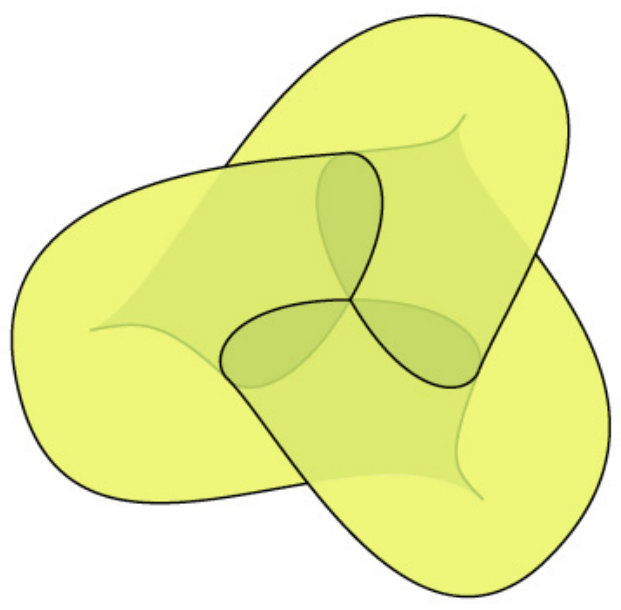

Figure 3. The Boy surface

Before showing how we can introduce a Boy surface into an arrangement of surfaces, we describe the atomic operations defined in [18]. The introduction of a Boy surface into a dual mesh will complete this set of operations. We use sheet diagrams [16] in the figures of this section. A dual mesh is an arrangement of surfaces which intersect each other. In a sheet diagram, each surface is represented by a sheet on which we can see how it is intersected by other sheets, that is surfaces. 


\subsection{Description of the Atomic Operations}

The atomic operations work in the dual of a hexahedral mesh which is a simple arrangement of surfaces. Formulating the problem in the dual allows us to take advantage of its combinatorial properties. Note that the corresponding primal-based operations could be defined, but they would be more difficult to understand and handle.

The ability to compute and visualize the hex mesh dual has been implemented in the qvdual tool, which is part of the MOAB package [10]. Shown in Figure 4 is a mesh, its dual surfaces, and a sheet diagram for one of those sheets. qvdual allows picking of dual 2- and 1-cells and implements the dual-based operations described here. qvdual uses the VTK toolkit for graphics rendering and the Qt package for the graphical user interface.

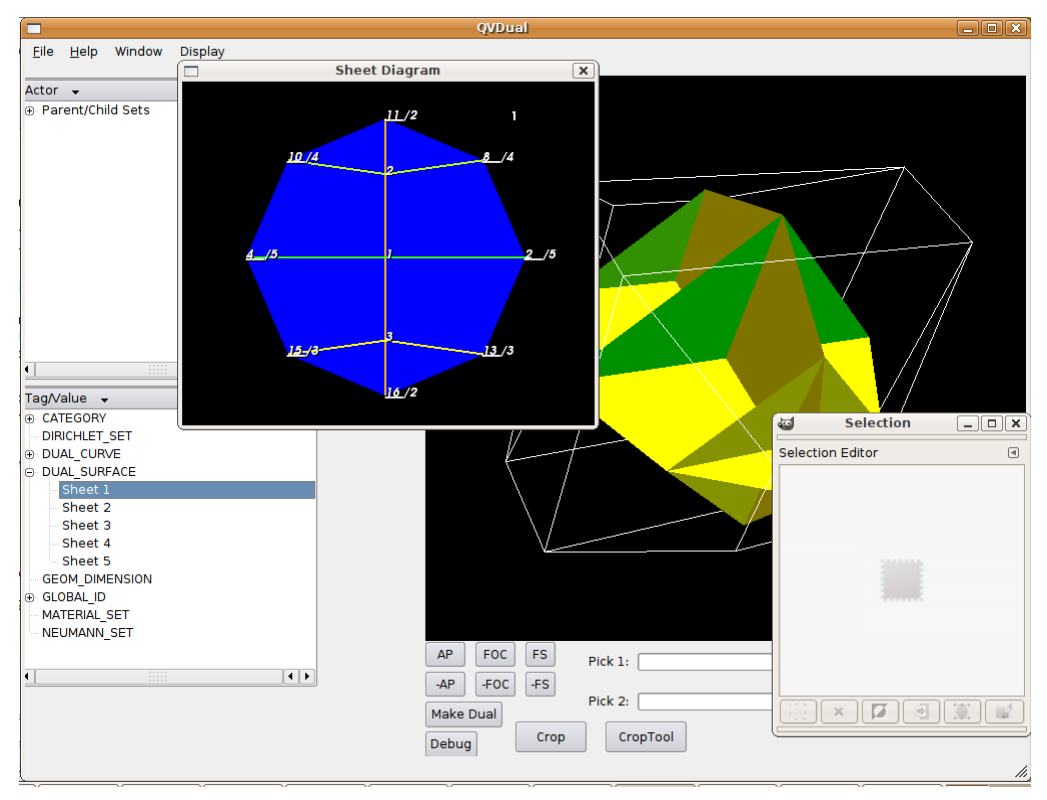

Figure 4. The qvdual

\subsubsection{Atomic Pillow}

In a pillowing operation, a new dual sheet is inserted in the dual arrangement such that it induces new vertices. In the primal mesh, it corresponds to adding a layer of hexes surrounding a local area in the mesh. The atomic pillow is the pillowing operation which adds the minimal number of vertices (respectively hexes) in the dual mesh (resp. primal mesh). This operation begins and ends with a conformal hexahedral primal mesh. Only two dual vertices (resp. hexes) are inserted in the dual mesh (resp. primal mesh) (see Figure 5). In the primal mesh, this operation pulls apart a quad, which separates two hexes, into two quads sharing all their edges. The void between these two quads is filled with two hexes sharing five quads together.

Figure 6 provides the sheet diagram corresponding to the Atomic Pillow. We can see that a new sheet is indeed inserted and it intersects only two others sheets.

\subsubsection{Face Shrink}

The face shrink operation consists in crossing two dual surfaces, i.e two sheets, along a dual 1-cell. By this way four dual 1-cell forming a circular line and four dual 0-cells are introduced in the dual mesh. On Figures 7 and 8, we can see how this operation acts. Each green sheet cross each other along the edge $A B$ and the edges $C D, D E, E F$ and $F G$ are created. 


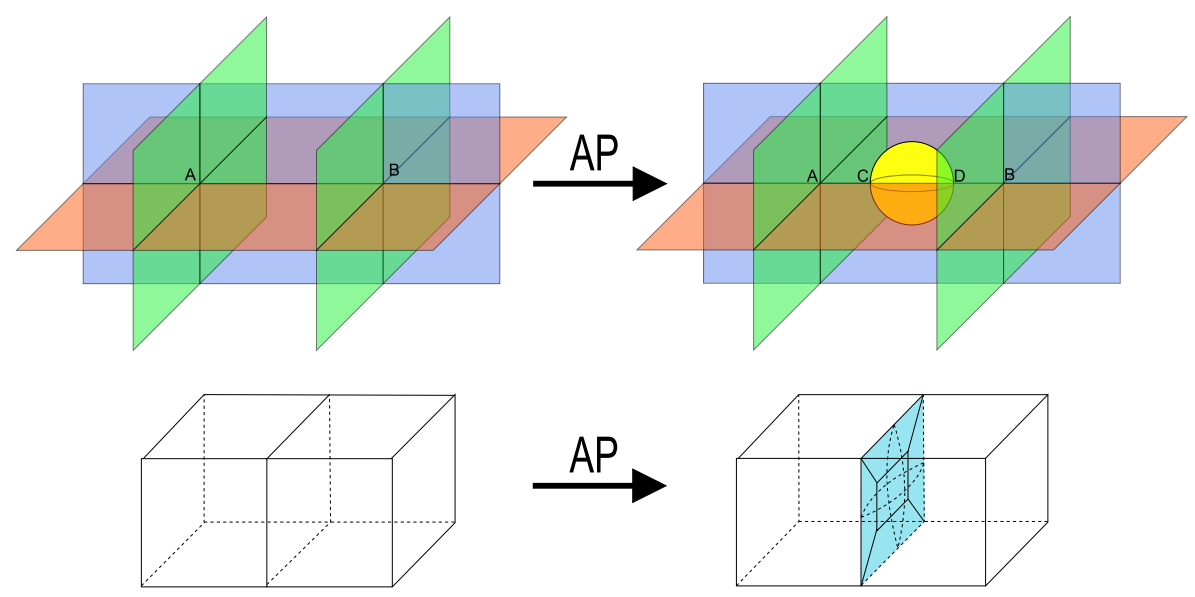

Figure 5. Atomic pillow in the dual mesh (up) and in the primal mesh (down)

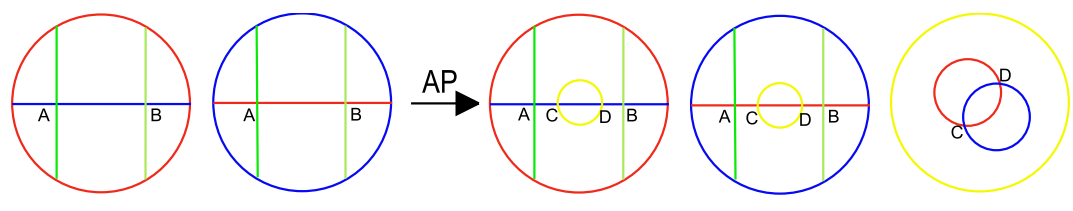

Figure 6. Atomic pillow in sheet diagrams

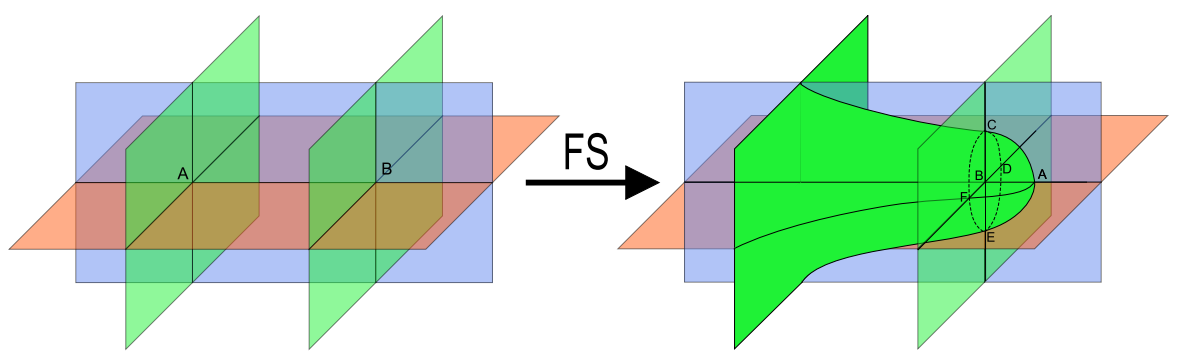

Figure 7. Face shrink in the dual mesh

In the dual mesh, two dual vertices are concerned by the operation ( $A$ and $B$ in our example). Thus, in the primal mesh, two hexes are concerned and the FS operation introduces four new hexes which makes a torus of hexes around the two initial hexes (see Figure 9).

\subsubsection{Face Open-Collapse}

The face open-collapse operation is a very tricky operation which is difficult to apprehend at first time. It consists in splitting and merging neighbouring dual surfaces. This operation modifies the cell complex locally without changing the boundary of the arrangement. Its name "face open-collapse" refers to how this operation modifies the primal mesh. 


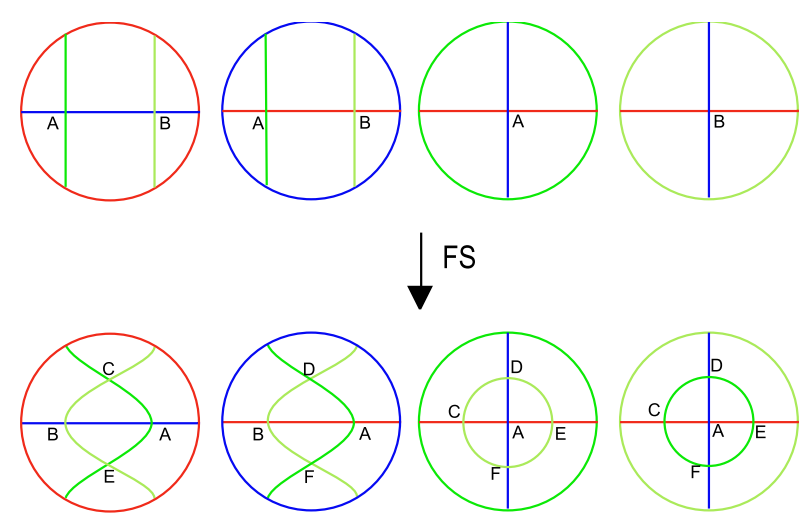

FiguRE 8. Face shrink in sheet diagrams

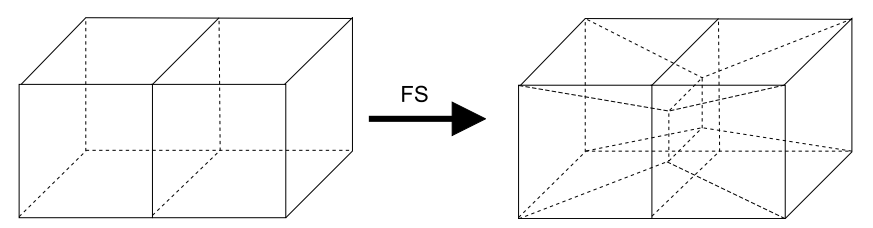

Figure 9. Face shrink in the primal mesh

The process of the FOC operation is the following. Two dual edges which bound a dual face are opened. For instance, on Figure 10, we open the edges $A B$ and $C D$ that bound the face $F$. We get then four dangling edges: $A x, B y, C z$ and $D w$. The dangling edges are then reconnected in such a way that the new edges do not intersect themselves. On Figure 10, the dangling edges $A x$ and $D w$ are connected and the dangling edges $B y$ and $C z$ are connected. In this way, two sheets are fused into one single sheet.

\subsection{Description of the Boy Surface Introduction into an Arrangement of Surfaces}

The principle is to put a copy of a Boy surface into a simply connected region of the dual mesh, which is an arrangement of surfaces, in such a way that the augmented arrangement of surfaces remains connected and continues to avoid multiple adjacencies. Each of the three loops of the Boy surface must cross the arrangement of surfaces an even number of times, so that the change of parity will be from the introduction of the single triple intersection of Boy surface with itself. To do that, we add the Boy Surface in the arrangement of surfaces defining the dual of an hexahedral mesh in such a way that each self-loop of the Boy surface intersects a different 2 -cell of the dual mesh. These three 2-cells must pass through one common vertex and intersect pairwise.

The introduction of a Boy surface into a dual mesh is presented through sheet diagrams. Figure 11 shows the diagram of the single sheet defining the Boy surface. We can see the three loops of the Boy surface, named $A, B$ and $C$. An edge in a sheet diagram represents the intersection between the represented sheet and another one. Thus, there are two "sheet edges" that correspond to a dual edge. Since, the Boy surface intersects itself, the two associated sheet edges occur on the same sheet diagram. For this reason, two edges are marked $A$, two others are marked $B$ and two others are marked $C$. The boundary of the sheet diagram is a square. In fact, opposite sides are connected. If an edge go out through the left side, it get in through the right one.

We intersect the Boy surface with three other sheets verifying the properties given previously. We get then the four sheet diagrams of Figure 12 and Figure 13. Figure 12 shows the Boy surface after the intersection with three other sheets. As expected, each sheet intersects only one self-loop of the Boy Surface. For instance, 


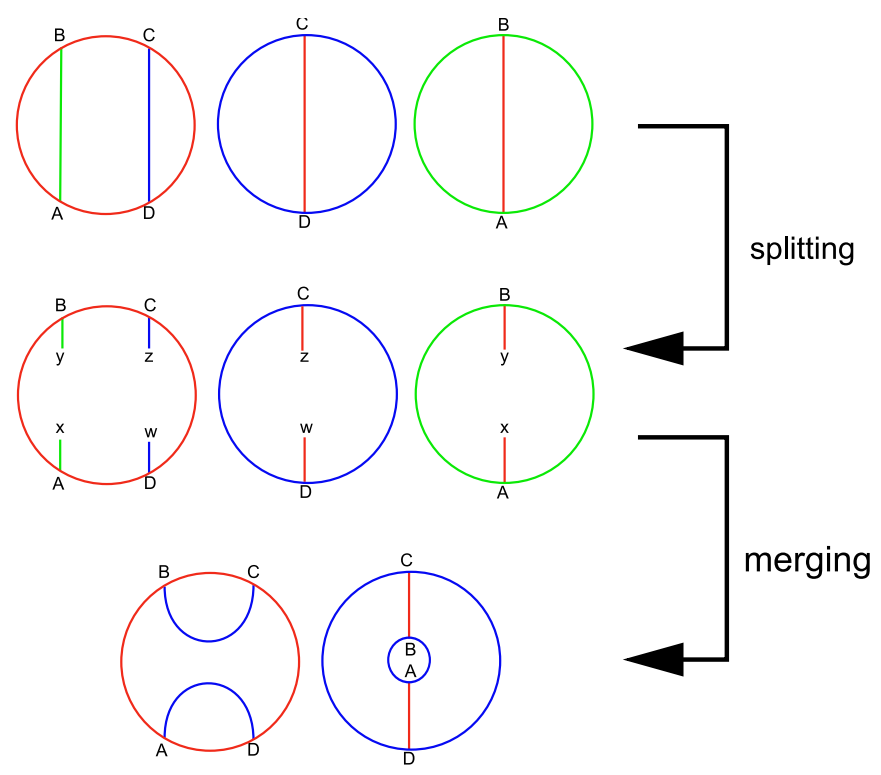

Figure 10. Face Open-Collapse with sheet diagrams

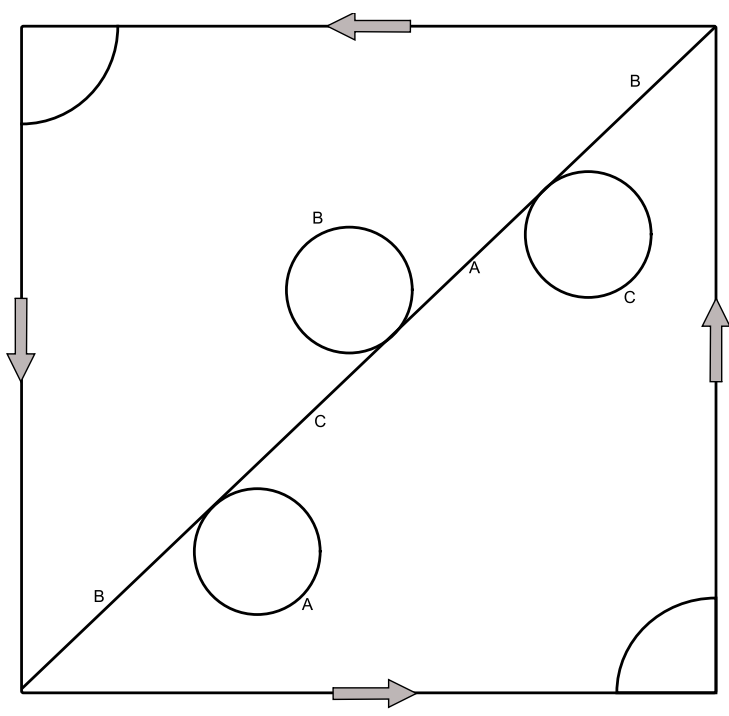

FiguRE 11. sheet diagram of a single Boy surface

the red sheet just cut the dual edge $B$ creating two vertices $a$ and $b$. Figure 13 shows the three sheets which intersect the Boy surface. They are symmetrical. Each sheet intersects the two other sheets twice.

\section{Conclusion}

In this paper, we dealt with the important and difficult issue of the local topological modifications in hexahedral meshes. First, we proved that the flipping operations defined by M. BERN and D. EppsteIN are 


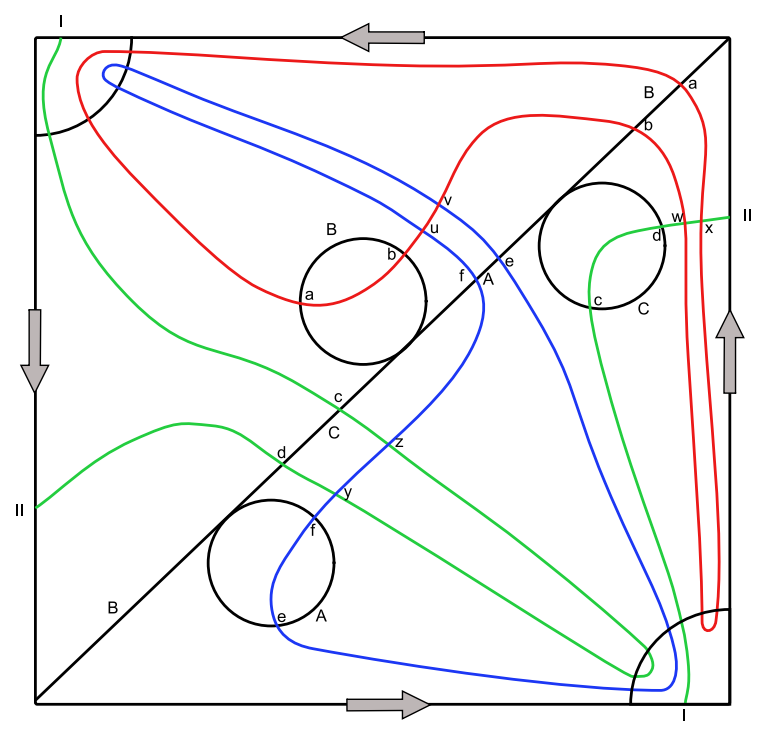

FiguRE 12. Intersection of the Boy surface with three pairwise orthogonal sheets
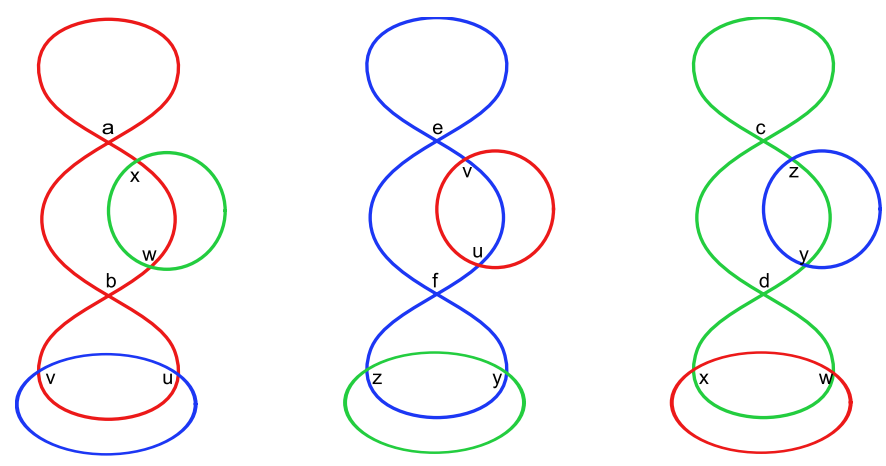

FIGURE 13. Sheet diagrams of the three sheets which intersect the Boy surface

combinatorially free. After that, we showed how to introduce a Boy surface into a dual mesh in order to modify the parity of the number of hexes in the primal mesh.

To go on with this work, it would be necessary to remove the self-loops and multiple adjacencies from the arrangement of surfaces obtained after the introduction of a Boy surface. This work is important to prove that the introduction of a Boy surface into a dual mesh could be an applyable fourth atomic operations. Then, it will remain to prove that the operations AP, FS, FOC with the introduction of a Boy surface, form a basis of the modifications of a hexahedral dual mesh. In other words, the combinatorial freedom of these four operations must be proved and we must show that any modification operation is a linear combination of these four operations. This theroretical work needs a deep knowledge in algebra and likely polytope theory.

\section{REFERENCES}

[1] M. Bern, D. Eppstein And J. Erickson: Flipping Cubical Meshes, ACM Computer Science Archive, http://www.arXiv.org/cs.CG/0108020, 2002.

[2] G. Blind And R. Blind: The almost simple cubical polytopes, Discrete Math., vol. 184, pages 25-48, 1998. 
[3] N.A. Calvo AND S.R. Idelsohn: All-hexahedral element meshing: Generation of the dual mesh by recurrent subdivision. Computer Methods in Applied Mechanics and Engineering, pages 371-378, 2000.

[4] S.A. Canann, S.N. Muthukrishann and R.K. Phillips: Topological refinement procedures for quadrilateral finite element meshes, Engineering with Computers, pages 168-177, vol. 12, 1998.

[5] N.T. Folwell And S.A. Mitchell: Reliable Whisker Weaving via Curve Contraction. 7th International Meshing Roundtable, pages 365-378, 1998.

[6] B. Grunbaum: Convex Polytopes. Interscience, London, 1967, 2nd Ed., V. Kaitel, V. Klee and G.M Ziegler, editors, Graduate Texts in Math., vol. 221, Springer-Verlag, New York, 2003.

[7] M. Joswig and G. M. Ziegler: Neighborly cubical polytopes, Discrete \& Computational Geometry arXiv:math.CO/9812033, vol. 24, pages 325-344, 2000.

[8] S. Matveev and M. Polyak: Cubic complexes and finite type invariants, arXiv:math.GT/0204085, 2000.

[9] M. Muller-Hannemann: Shelling Hexahedral Complexes for Mesh Generation. Journal of Graph Algorithms and Applications, $5(5)$, pages 59-91, 2001.

[10] HTTP://TRAC.MCS.ANL.GOV/PROJECTS/ITAPS/WIKI/MOAB.

[11] P. Murdoch and S.E. Benzley: The Spatial Twist Continuum: A Connectivity Based Method for Representing All Hexahedral Finite Element Meshes, 4th International Meshing Roundtable, SAND95-2130, Sandia National Laboratories, Albuquerque, 1995.

[12] A. Schwartz: Constructions of Cubical Polytopes. Phd Dissertation, TU Berlin, $2003 . \quad$ Available at http://edocs.tu-berlin.de/diss/2004/schwartz_alexander.html.

[13] M.B. Stephenson, S.A. Canann and T.D. Blacker: Plastering: a new approach to automated 3D hexahedral mesh generation. Progress Report SAND89-219, Sandia National Laboratories, 1992.

[14] T.J. Tautges: A set of atomic, dual-based operations for modifying hexahedral mesh topology. Presentation to UW-madison Math. Dept., Topology Group, 2004.

[15] T.J. TAutges: Local Topological Modification of Hexahedral Meshes Using Dual-Based Operations, 8th U.S . National Conference on Computational Mathematics, Austin, TX, July 2005.

[16] T.J. Tautges, T.D. Blacker and S.A. Mitchell: The Whisker Weaving Algorithm: A Connectivity-based Method for Constructing All-hexahedral Finite Element Meshes. International Journal For Numerical Methods in Engineering, vol. 39, pages 3327-3349, 1996.

[17] T.J. Tautges and S.E. Knoop: Topology Modification of Hexahedral Meshes Using Atomic Dual-Based Operations, 12th International Meshing Roundtable, pages 415-423, 2003.

[18] T.J. Tautges, S.E. Knoop and T.J. Rickmeyer: Local Topological Modification of Hexahedral Meshes; Part I: A Set of Dual-Based Operations, ESAIM Proc., vol. 24, p. 14-33, 2008.

[19] G.M. Ziegler: Lectures on Polytopes. Graduate Texts in Math., vol. 152, Springer-Verlag, New York, 1995, revised ed., 1998. Updates, corrections, etc. available at http://www.math.tu-berlin.de/ ziegler. 